\title{
Evidence for a diffusion-based mechanism of liquid metal intergranular penetration : case study of a Ni-Bi model system
}

\author{
K. Wolski ${ }^{1, a}$, N. Marie ${ }^{1, b}$, V. Laporte ${ }^{1, c}$, P. Berger ${ }^{2, d}$ and M. Biscondi ${ }^{1, e}$ \\ ${ }^{1}$ Ecole des Mines de St-Etienne - UMR CNRS 5146 PECM 158 c. Fauriel F-42023 Saint-Etienne - FRANCE \\ ${ }^{2}$ CEA / SACLAY DEN / DPC / SCCME F-91191 Gif Sur Yvette Cedex - FRANCE \\ ㅁwolski@emse.fr, ${ }^{\mathrm{b}}$ marie@emse.fr, ${ }^{\mathrm{C} l a p o r t e @ e m s e . f r},{ }^{\mathrm{d}}$ pascal.berger@cea.fr, ${ }^{\mathrm{e} b i s c o n d i @ e m s e . f r}$
}

Keywords: Ni-Bi system, IGP - intergranular penetration, grain boundary wetting, intergranular diffusion, Fowler-Guggenheim segregation isotherm, AES - Auger electron spectroscopy, spallation target, LME - liquid metal embrittlement.

\begin{abstract}
A model $\mathrm{Ni}-\mathrm{Bi}$ system has been used to investigate intergranular penetration (IGP) phenomenon. All experiments have been done on $\mathrm{Ni} 26^{\circ}<110>$ bicrystal at $700^{\circ} \mathrm{C}$ using bismuth vapour condensation as a source of liquid bismuth. Such a procedure results at room temperature in either partial or total Liquid Metal Induced Embrittlement (LMIE) of a unique grain boundary, depending on the duration of liquid $\mathrm{Bi} /$ solid Ni contact at $700^{\circ} \mathrm{C}$. Auger Electron Spectrometry (AES) and Rutherford Backscattering Spectrometry (RBS) have been used to measure the Bi concentration profile between the source of liquid bismuth and the penetration front. Two zones have been clearly identified : the first one of almost constant $\mathrm{Bi}$ concentration called nanometrethick film which is interpreted in terms of Fowler-Guggenheim multi-layer segregation under local equilibrium conditions and the second one with a progressive decrease of Bi concentration over a distance of the order of $20-200 \mu \mathrm{m}$. Such a long transition zone, together with parabolic diffusion kinetics indicates diffusion-based mechanism of intergranular penetration as opposed to the direct grain boundary wetting.
\end{abstract}

\section{Introduction}

Intergranular penetration (IGP) is the replacement of solid metal grain boundaries by a liquid metal that occurs without any applied stress and results in the formation of either micrometre-thick or nanometre-thick films [1]. Such a replacement is possible if the energy of solid metal grain boundaries is higher than the double of the solid metal / liquid metal interfacial energy [2]. This currently used definition describes only the result of the thermodynamic equilibrium and, consequently, there is no indication neither of the mechanism nor of the kinetics of this phenomenon.

IGP might be critical in the long-term behaviour of a spallation target, where T-91 steel has to withstand the contact with liquid $\mathrm{Pb}-\mathrm{Bi}$ eutectic alloy [3]. IGP can potentially lead to two modes of damage : first, liquid metal embrittlement (LME) at high temperature under simultaneous action of stress and liquid metal and, second, liquid metal induced embrittlement (LMIE) typically under the action of stress at room temperature when liquid metal has solidified.

The prediction of the long-term behaviour of structural components under such conditions requires the knowledge of the mechanism of intergranular penetration. This is the main reason why we decided to study this phenomenon using a model solid Ni / liquid Bi system, in which IGP is known to occur at $700^{\circ} \mathrm{C}$ [4]. There are at least three advantages to use this model system : first IGP occurs in only a few hours (as compared to several months in systems of industrial interest), second, it results in LMIE and therefore gives access to the depth of embrittlement and finally it offers the possibility to analyse the bismuth composition profile on the fracture surface either by Auger Electron Spectrometry (AES) after "in situ" fracture or by Rutherford Backscattering Spectrometry (RBS) even after ex situ fractures. The main drawbacks are the existence of a peritectic 
transformation at $646^{\circ} \mathrm{C}$ and the significant volume diffusion above $750^{\circ} \mathrm{C}$, both limiting the investigation of IGP to the temperature of $700^{\circ} \mathrm{C}$.

It has been previously shown that the direct contact between liquid Bi-rich alloy and solid $\mathrm{Ni}$ at $700^{\circ} \mathrm{C}$ results in the formation of short micrometre-thick films that replaces typical Mullins grooves [5] and ahead, long nanometre-thick films that have to be considered with respect to both LMIE and LME, because they result in similar degree of embrittlement and develop much faster than micrometre-thick films [6]. In particular, the precise evaluation of the bismuth concentration profile at the tip of this nanometre-thick film is critical with respect to the mechanism of IGP. The question which arises from this analysis is to know what is the characteristic distance where original pure grain boundary is replaced by a nanometre-thick film : is it of the order of some interatomic distances up to some nanometres ? or, on the contrary, is it a much longer distance of the order of several microns? While the former (very short transition) would be representative of a wettingbased mechanism [7], the latter would clearly indicate the diffusion-based mechanism of IGP ?[8]. The purpose of this presentation is to provide new experimental data on the Ni-Bi system, concerning first, the bismuth transition profile as analysed by AES and RBS, second, the kinetics of IGP and finally, to discuss these results with respect to the possible mechanism of IGP.

\section{Experimental conditions}

$\mathrm{Ni}$ bicrystals with a general $26^{\circ}$ symmetrical tilt grain boundary around [110] axis have been used. All bicrystals have been cut by spark erosion as approximately (1 to 2) x (2 to 3$) \times 18 \mathrm{~mm}^{3}$ parallelepipeds and subsequently electrochemically polished in order to avoid any re-crystallization during subsequent heat treatments. All heat treatments were done at $700^{\circ} \mathrm{C}$ in presence of $\mathrm{Bi}$ vapours and, due to its high partial pressure, resulted in the deposition of a thin liquid Bi film. It has been previously shown that such a $10 \mathrm{~nm}$ thick film, as analysed by GDOS analysis, is equivalent to the action of a bulk Bi-rich liquid in equilibrium with $\mathrm{Ni}$ at $700^{\circ} \mathrm{C}$ [9].

Two bicrystals have been heat treated for $15 \mathrm{~min}$ and $22 \mathrm{~min}$ in order to obtain partial penetration, then introduced to the main chamber of the AES, "in situ" broken respectively by tensile test and by impact bending, and subsequently analysed on the as revealed fracture surfaces in order to access to the intergranular bismuth penetration profile. One additional bicrystal $\left(1 \mathrm{~h}\right.$ at $700^{\circ} \mathrm{C}$, slowly cooled to the room temperature) has been analysed by RBS spectrometry.

Then a series of bicrystals have been treated for $0.5,1,1.5,2$ and 2.5 hours and subsequently tensile tested at room temperature in order to reveal the depth of embrittlement and in this way to access the kinetics of intergranular bismuth penetration. Additionally, a series of polycrystals have been heat-treated at $700^{\circ} \mathrm{C}$ for $0.25,0.5,1$ and 2 hours in order to generalise the kinetics obtained on the $26^{\circ}$ bicrystal. In the latter series, intergranular penetration depth was assimilated to the mean crack length as determined from the observation of longitudinal polished cross sections after $20 \%$ prestraining at room temperature.

\section{Bismuth concentration profiles}

The $26^{\circ}<110>$ bicrystal heat treated without any applied stress for $15 \mathrm{~min}$ at $700^{\circ} \mathrm{C}$ has been broken at room temperature by tensile test within the main chamber of the AES. The resulting fracture (Fig. 1) is the consequence of a partial intergranular penetration at $700^{\circ} \mathrm{C}$. The outer brittle ring corresponds to the area penetrated by bismuth. The inner ductile part represents the behaviour of solid nickel unaffected by bismuth penetration. Fig. 2 presents the detail of the transition from the brittle region, which is flat and smooth (a), through the transition zone where micro-facetting was systematically present over a distance of the order of $100 \mu \mathrm{m}$ (b), up to the ductile core (c). The width of the micro-facetted transition zone varied from 20 to $200 \mu \mathrm{m}$ even along the same edge of the specimen. These general trends have been confirmed by the observation of the specimen after $22 \mathrm{~min}$ heat treatment at $700^{\circ} \mathrm{C}$. 
AES point-to-point analysis every $50 \mu \mathrm{m}$ has been done along the AB line in Fig. 1. At each point, AES spectra was recorded and analysed. Only Bi and Ni peaks were present. The amount of bismuth is represented by the $\mathrm{Bi}$ to $\mathrm{Ni}$ ratio, defined as the area of the $100 \mathrm{eV}$ double $\mathrm{Bi}$ peak divided by the area of $845 \mathrm{eV}$ single Ni peak [5]. This way of representation was chosen in order to insist on the relative change in the amount of $\mathrm{Bi}$, especially in the transition zone (cf. Fig.2b).

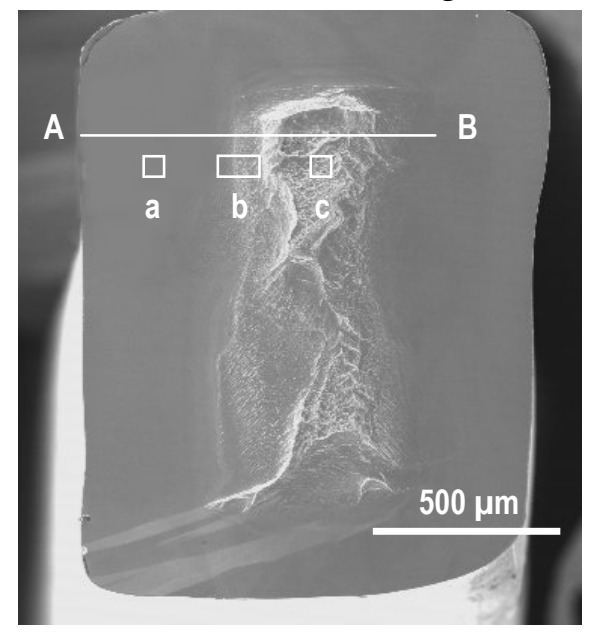

Fig. 1. Fracture surface of $26^{\circ}<110>$ bicrystal partially embrittled due to the intergranular Bi penetration (15 min at $\left.700^{\circ} \mathrm{C}\right)$.
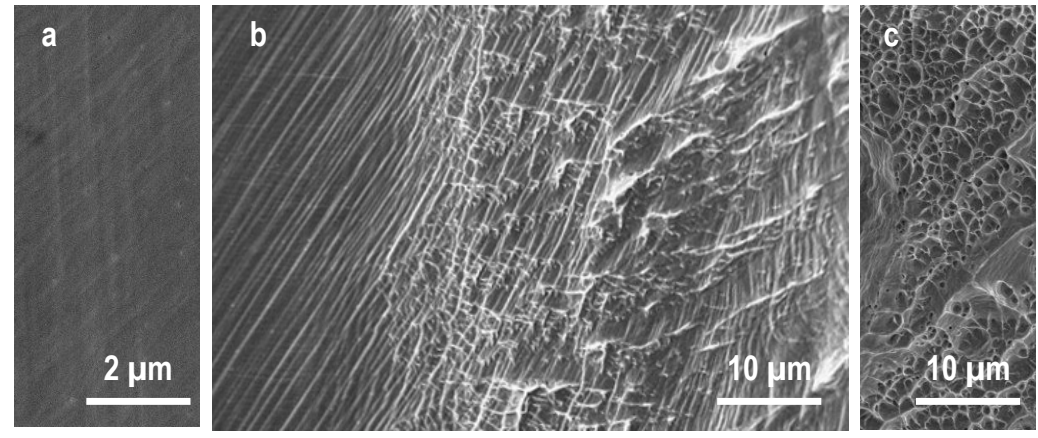

Fig. 2. Detailed view of the fracture surface from Fig.1 indicating: a. almost flat fracture surface in the brittle region, b. transition zone between this brittle region and $c$. ductile core.

Fig. 3 represents the evolution of the $\mathrm{Bi}$ to $\mathrm{Ni}$ ratio as a function of the distance from the point $\mathrm{A}$ at the edge of the specimen towards the point B (cf. Fig.1). The amount of bismuth is almost constant over a distance of approximately $250 \mu \mathrm{m}$ and again between 850 and $950 \mu \mathrm{m}$, i.e. in two brittle regions (cf. Fig. 2a) in the outer ring of the fracture surface. In the area corresponding to the ductile inner core of the specimen, between 400 and $600 \mu \mathrm{m}$, no bismuth was detected. The most important feature in this figure is the progressive decrease of the amount of bismuth between 250 and $400 \mu \mathrm{m}$ and between 800 and $600 \mu \mathrm{m}$, both corresponding to the micro-facetted regions of the fracture surface. Once again similar evolution was observed on the $26^{\circ}<110>$ bicrystal heat treated for $22 \mathrm{~min}$ at $700^{\circ} \mathrm{C}$. A clear correlation between the width of the micro-facetted region and the sharpness of the bismuth concentration profile was observed : the narrower the micro-facetted transition zone, the faster the decrease of the Bi to Ni ratio.

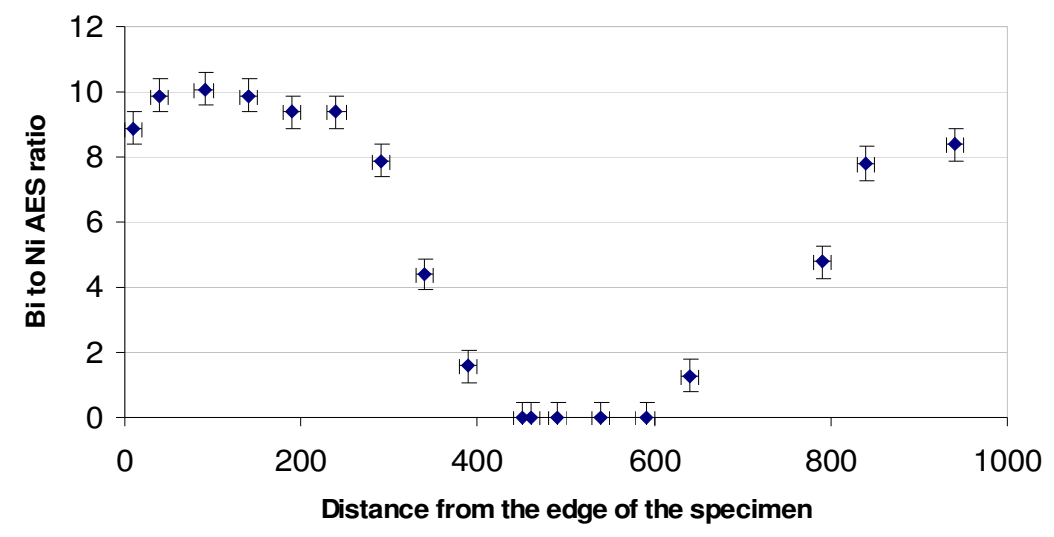

Fig. 3. Evolution of the Bi to Ni intensities ratio along the line $A B$ indicated in Fig.1, as measured by $A E S$ on the $26^{\circ}<110>$ bicrystal $\left(15 \mathrm{~min}, 700^{\circ} \mathrm{C}\right)$.

Microspot RBS with the spatial resolution of one micrometer has been used to measure the evolution of the amount of bismuth across a narrow transition zone in the specimen heat treated for $1 \mathrm{~h}$ at $700^{\circ} \mathrm{C}$ and slowly cooled to room temperature (such a procedure activates sulphur segregation induced brittleness and, due to the substantial reduction in the micro-roughness, increases the accuracy of RBS analysis). A suitable transition zone has been localised after "ex-situ" fracture by 
SEM observations and subsequently, a $100 \mu \mathrm{m} \times 100 \mu \mathrm{m}$ area was analysed by RBS. Fig. 4 represents a fragment of the fracture surface between the brittle outer region and the "ductile" inner core (in fact here partially brittle due to the sulphur segregation) together with (i) the black square, which marks the exact area analysed by RBS and (ii) the dotted white rectangle, which indicates the transition zone. Within this zone the absolute Bi signal was extracted from $10 \mu \mathrm{m}$ wide vertical bands positioned every 10 or $5 \mu \mathrm{m}$ and has allowed to give the evolution of the absolute amount of $\mathrm{Bi}$ as a function of the distance, as indicated in Fig. 5. It clearly appears that the width of this "narrow" transition zone is in this case of the order of $30 \mu \mathrm{m}$. All AES and RBS analysis together with fractographic observations indicate that the width of the transition zone keeps within the range of 20 up to $200 \mu \mathrm{m}$.

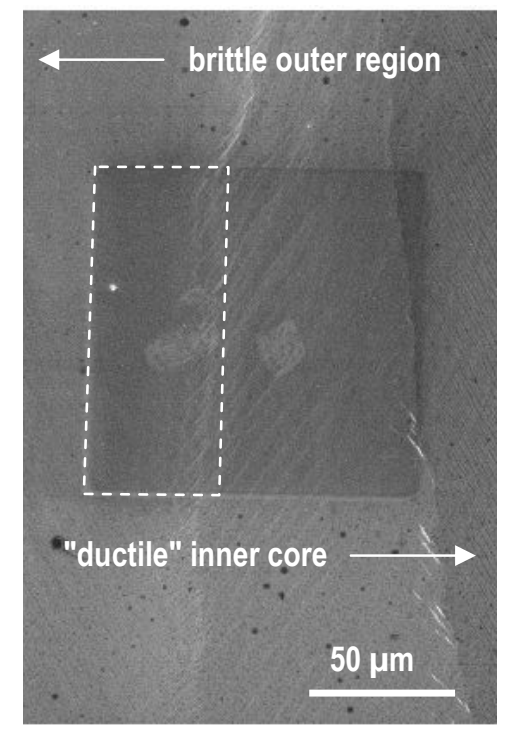

Fig. 4. Fracture surface of the $\left(1 \mathrm{~h}, 700^{\circ} \mathrm{C}\right)$ sample analysed by RBS.

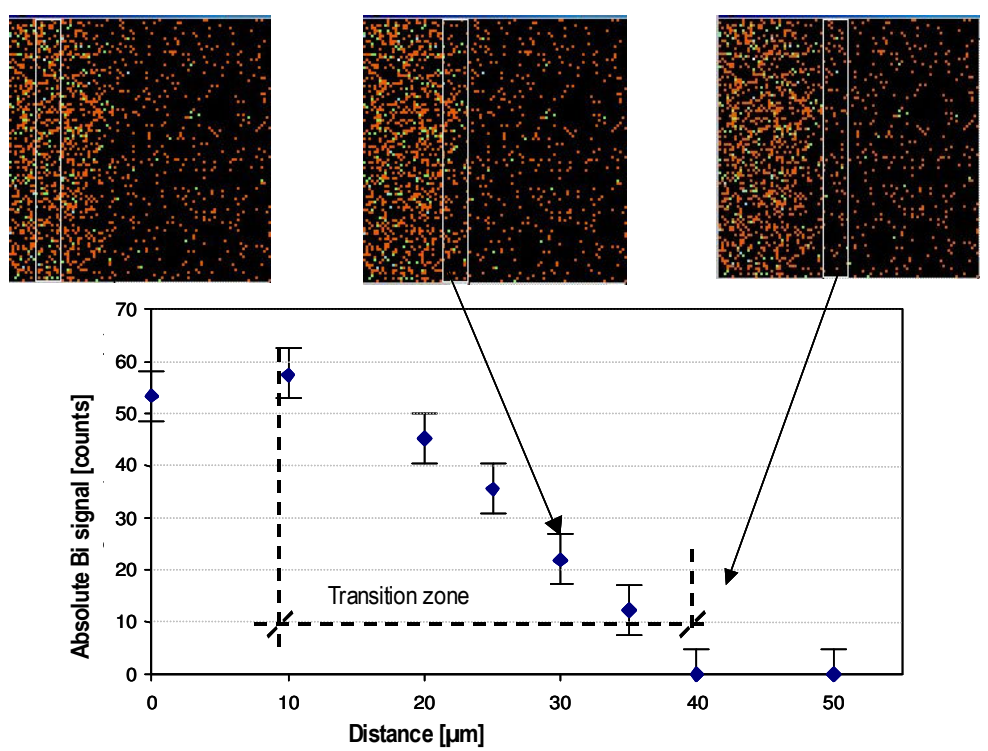

Fig. 5. Evolution of the absolute Bi signal across the transition zone, as measured by RBS on the $26^{\circ}<110>$ bicrystal $\left(1 \mathrm{~h}, 700^{\circ} \mathrm{C}\right)$.

\section{Kinetics of bismuth penetration}

It is important to once again clearly distinguish between two phenomena : first, intergranular penetration which occurs at $700^{\circ} \mathrm{C}$ without any external stress and results in progressive incorporation of $\mathrm{Bi}$ atoms into $\mathrm{Ni}$ grain boundaries and second, liquid metal induced embrittlement (LMIE) which occurs at room temperature under the influence of external stress and results in the brittle intergranular fracture from external surface close to the tip of penetrating $\mathrm{Bi}$ atoms. In this study LMIE is only a way to reveal the depth of IGP. In order to measure the kinetics of intergranular penetration, we are going to associate the depth of $\mathrm{Bi}$-induced intergranular embrittlement to the depth of intergranular penetration, because the length of a Bi tail at the very tip (ahead of brittle region) can be neglected as compared to the length of the overall embrittled area.

The mean depth of embrittlement on $\mathrm{Ni} 26^{\circ}<110>$ bicrystals as a function of square root of time of heat treatment under $\mathrm{Bi}$ vapours at $700^{\circ} \mathrm{C}$ is represented in Fig. 6. Each pair of points, for a given time, corresponds to the penetration depth in the direction either parallel or perpendicular to the tilt axis of the bicrystal. In both cases straight lines have been obtained, which is indicative of parabolic penetration kinetics. Such a kinetics is typical of a $\mathrm{C}$ type diffusion in a classical Harrison classification [10]. In the present case it is compatible with a very limited solubility of Bi in solid $\mathrm{Ni}$ at $700^{\circ} \mathrm{C}$ (never specified on phase diagrams, therefore supposed to be lower than $10 \mathrm{ppm}$ ). Note also that the observed anisotropy (penetration faster in the perpendicular direction) is surprising with respect to the classical models of low-angle grain boundaries, yet it has been observed in the past on high-angle general grain boundaries, where fast diffusion pipes in the parallel direction are no longer efficient with respect to the grain boundary structure in the perpendicular direction [11]. 


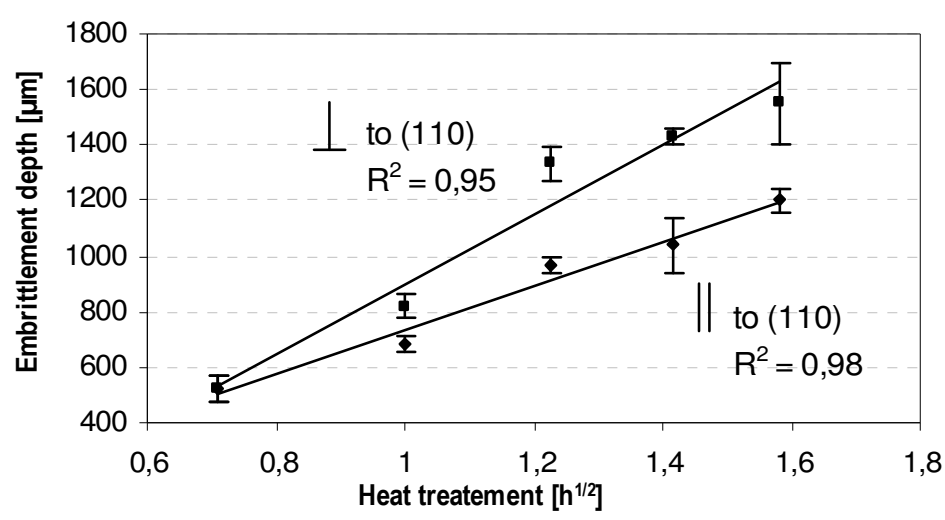

Fig. 6. Kinetics of grain boundary embrittlement of Ni bicrystal at $700^{\circ} \mathrm{C}$ along two directions, respectively perpendicular and parallel to the (110) tilt axis.

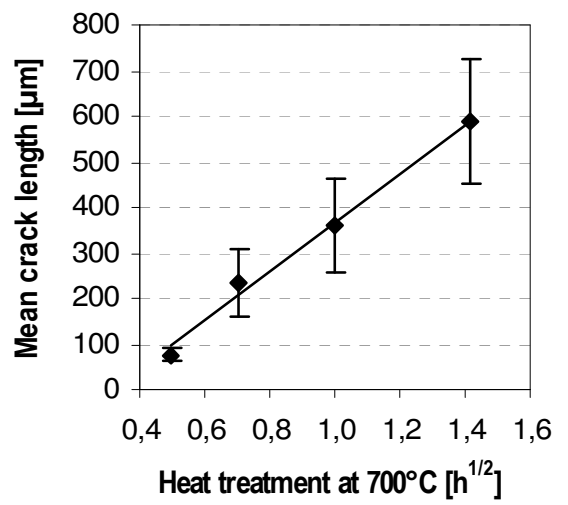

Fig. 7. Kinetics of grain boundary embrittlement in Ni polycrystals at $700^{\circ} \mathrm{C}$.

The kinetics of intergranular penetration was also analysed on a series of Ni polycrystals. Fig. 7 represents mean crack length as a function of the square root of time of heat treatment under $\mathrm{Bi}$ vapours at $700^{\circ} \mathrm{C}$. The important uncertainty in this series comes from a wide distribution of grain boundary types and orientations with respect to the tensile axis, which is typical for any polycrystalline material. Yet, Fig. 7 confirms the parabolic kinetics obtained from the rigorous analysis of a specific $26^{\circ}<110>$ bicrystal (cf. Fig. 6).

\section{Indications for a diffusion-based mechanism of IGP}

The Bi concentration profile obtained in this study by two independent spectroscopic measurements (AES and RBS) and schematised in Fig. 8, clearly indicates that the transition from the pure grain boundary (between points $\mathrm{F}$ and $\mathrm{E}$ in Fig. 8) to the grain boundary saturated with Bi atoms (B-A, in Fig. 8) occurs on the distance within the range $20-200 \mu \mathrm{m}$ (D-B in Fig. 8), after heat treatments up to one hour at $700^{\circ} \mathrm{C}$. Such a long distance is incompatible with any IGP model based on direct grain boundary wetting, where the transition is supposed to occur on the distance of the order of some nanometres, i.e. four orders of magnitude less than in the present case.

The area of almost constant $\mathrm{Bi}$ concentration (B-A, in Fig. 8) corresponds to the saturated grain boundary. The level of saturation was previously estimated from AES quantitative measurements to be within the range 2-3 $\mathrm{nm}$ [12] and consequently the existence of nanometre-thick films was postulated in the present system. The formation of such films can be explained on the basis of the Fowler-Guggenheim segregation isotherm together with local equilibrium between grain boundary concentration and very limited bulk Bi solubility, using the formalism proposed in the case of $\mathrm{Cu}-\mathrm{Bi}$ system [13].

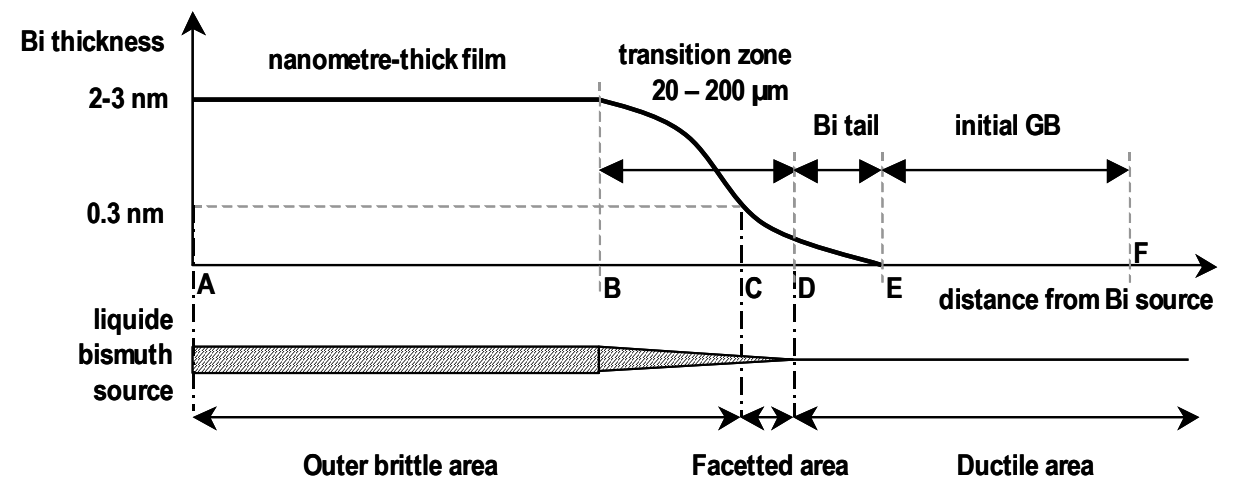

Fig. 8.

Schematic representation of bismuth concentration profile along penetrated grain boundary, together with the specific vocabulary used in this paper and indications about the type of fracture. The $20-200$ $\mu \mathrm{m}$ transition zone covers both the facetted area and a part of the brittle area. Bi tail is a part of grain boundary where $\mathrm{Bi}$ has diffused, but its amount is under the detection limit. 
The commonly admitted view of grain boundary penetration is that this is a phenomenon which occurs in some specific systems within the range of temperatures where grain boundary energy is higher than twice the solid metal / liquid metal interfacial energy [14]. This phenomenon is supposed to occur on a very short distance $[7,15]$ with linear kinetics $[16,17]$ and often results in the formation of nanometre-thick films $[5,18,19]$.

While nanometre-thick films were effectively observed in several systems, including $\mathrm{Ni}-\mathrm{Bi}$, the existence of such a long transition profile together with the parabolic kinetics measured on both bicrystals and polycrystals, indicate a diffusion-based mechanism for the intergranular penetration phenomenon.

It appears from this study that the oversimplified view of IGP as direct replacement of initial grain boundary by a liquid metal film, occurs in reality by a two-steps process, first intergranular diffusion with a parabolic kinetics and second grain boundary saturation, which can be interpreted in terms of Fowler-Guggenheim multi-layer segregation resulting in the formation of nanometrethick intergranular films.

\section{Conclusions}

1. Bi-concentration profile in $\mathrm{Ni}-\mathrm{Bi}$ system at $700^{\circ} \mathrm{C}$, obtained on $26^{\circ}<110>\mathrm{Ni}$ bicrystal by two independent spectroscopic methods AES and RBS indicates the existence of a long 20-200 $\mu \mathrm{m}$ transition zone between initial grain-boundary and nanometre-thick films.

2. The overall profile can be interpreted in terms of Fowler-Guggenheim multiplayer segregation.

3. The kinetics of intergranular penetration is clearly parabolic on both bicrystals and polycrystals.

4. The results of this study give a clear evidence for a diffusion-based mechanism of IGP of liquid bismuth into solid nickel at $700^{\circ} \mathrm{C}$, as opposed to the direct grain boundary wetting.

\section{References}

[1] W. Rostoker, J. M. McCaughey, and H. Markus, Embrittlement by Liquid Metals, Reinhold Publishing Corp., New York, (1960).

[2] N. Eustathopoulos, Int. Metals Rev., 24 [4] (1983) 189-210.

[3] G. S. Bauer, M. Salvatores, and G. Heusener, J. of Nucl. Mat., 296 (2001) 17-33.

[4] G. H. Bishop, Trans. AIME, 242 (1968) 1343-1351.

[5] N. Marié, K. Wolski, and M. Biscondi, Scripta Mat., 43 (2000) 943-949.

[6] K. Wolski, V. Laporte, N. Marié, and M. Biscondi, Interf. Sci., 9 (2001) 183-189.

[7] E. Rabkin, in Multiscale phenomena in plasticity : from experiments to phenomenology, modeling \& materials engineering, edited by J. Lépinoux, D. Mazière, V. Pontikis, and G. Saada, Ouranoupolis, Greece. Kluwer Academic Publishers (1999) 403-413.

[8] H. J. Vogel and L. Ratke, Acta Metall. Mater., 39 [4] (1991) 641-649.

[9] N. Marié, K. Wolski, and M. Biscondi, J. of Nucl. Mat., 296 (2001) 282-288.

[10] Y. Mishin, C. Herzig, J. Bernardini, and W. Gust, Int. Mat. Rev., 42 [4] (1997) 155-178.

[11] I. Herbeuval, M. Biscondi, and C. Goux, Rev. de Mét., LXX [1] (1973) 39-46.

[12] K. Wolski, N. Marié, and M. Biscondi, Surf. Interf. An., 31 (2001) 280-286.

[13] V. Laporte, K. Wolski, P. Berger, A. Terlain, and G. Santarini, in DIMAT'2004, Krakow, Poland (2004) in this proceedings.

[14] B. Straumal and W. Gust, J. of Phase Eq., 15 [4] (1994) 386-391.

[15] E. E. Glickman, Mat. Sci. Forum, 294-296 (1999) 405-410.

[16] E. E. Glickman and M. Nathan, J. Appl. Phys., 85 (1999) 3185-3191.

[17] B. Joseph, F. Barbier, and M. Aucouturier, Mat. Sci. Forum, 294-296 (1999) 735-738.

[18] E. Rabkin and W. Gust, in Ceramic Interfaces - properties and applications, edited by R. Smart and J. Nowotny. IOM Communications Ltd, London, (1998) 151-170.

[19] B. Straumal and W. Gust, Mat. Sci. Forum, 207-209 (1996) 59-68. 